\title{
Continuous Peripheral Nerve Catheters in Pediatric Complex Regional Pain Syndrome
}

\author{
Helen Gharaei ${ }^{1{ }^{*}}$ \\ ${ }^{1}$ Department of Anesthesiology and Pain Medicine, Boali Hospital, Tehran Medical Branch, Islamic Azad University, Tehran, IR Iran \\ ${ }^{*}$ Corresponding author: Helen Gharaei, Department of Anesthesiology and Pain Medicine, Boali Hospital, Tehran Medical Branch, Islamic Azad University, Tehran, IR Iran. Tel: +98- \\ 2133348036, Fax:+98-2133348036, E-mail: helengharaee@yahoo.com
}

Received: September 7, 2014; Revised: October 12, 2014; Accepted: October 19, 2014

Keywords:CRPS (Complex Regional Pain Syndromes); Nerve Block; Catheters

Complex regional pain syndrome (CRPS) is outlined as a painful and disabling syndrome accompanied by physical changes within the affected extremity, together with allodynia, edema, and baldness, yet as sudomotor and dilatation dysfunction CRPS has been reported most often in adult population, but it has been reported in pediatric population as well. Although several modalities are tried for the treatment of CRPS, given the debilitating nature of disorder and variable response to medical aid, new methods of treatment are needed. Continuous peripheral nerve catheters reduce pain and facilitate intensive physiotherapy and practical rehabilitation, they resulted in resolution of physical changes associated with CRPS and a decrease need for pharmacological drugs, including opioids. Although they are not first-line treatment within the care of youngsters with CRPS.

Complex regional pain syndrome (CRPS) is outlined as a painful and disabling syndrome accompanied by physical changes within the affected extremity, together with allodynia, edema, and baldness, yet as sudomotor and dilatation dysfunction. In CRPS type I (reflex sympathetic dystrophy), small injuries or fractures of a limb initiate the onset of symptoms. CRPS type II (causalgia) develops once injury to a large peripheral nerve happened (1). Though reported most often in adult population, it has been recognized frequently in pediatric population. CRPS in kids is completely different from adults, there seem to be comparatively few variations (2). Recurrence rates seem beyond in adults; however, the response to reinitiation of treatment appears to proceed expeditiously. Diagnosis of CRPS is possible by findings from patient's history and physical examination. Pain, significantly with allodynia, and signs of autonomic instability either traditionally or on examination are needed to form this diagnosis. The pain ought to be out of proportion to the inciting event and sometimes distally generalized within the extremity. Pathologic processes that may produce pain should be excluded. There is no laboratory test to completely diagnose or exclude this diagnosis. Sporadic early reports of youngsters with CRPS appeared within the 1970s. Many of those patients had spontaneous resolution. This led to the suggestion that no treatment ought to be performed for youngsters with CRPS. The explanation was that each treatment carries risks and side effects. Recurrence occurred in more or less than 30\% to $50 \%$ of patients, a far higher rate than the speed of $1.8 \%$ per patient-year reported for adults. Though recurrence is common, it seems necessary to consider physiotherapy (PT) and connected treatments in the first episode (3).

Various treatment regimens are used including PT, behavioral measures, transcutaneous electrical nerve stimulation, antidepressants or anticonvulsants and regional anesthetic techniques, neuraxial blockade, Bier block, peripheral nerve catheters or sympathetic blockade (46 ). Intense exercise therapy is critical to treat childhood CRPS effectively and to decrease the high incidence of recurrence reported in patients treated solely by PT and cognitive-behavioral treatment. However, PT is incredibly painful throughout CRPS and a method should be coordinated with PT sessions to relieve pain of patients throughout the sessions. Regarding anesthesia, numerous techniques are represented in adult literature. However, no specific intervention has shown to be superior in treating or preventing the signs and symptoms of CRPS. Though the pathophysiology is not fully perceived, CRPS is presently felt to be a derangement in the central process ensuing from altered neuroplasticity. Peripheral nerve blockade treats the endpoint symptoms, however cannot suppress activity at the dorsal root ganglion and does not address the chronic sensitization within the disease process. Sympathetic blockade is also useful in some cases (1). Sympathetic blocks might facilitate outline the proportion of pain sympathetically mediated at that point and would be of therapeutic profit, however

Copyright (C) 2015, Iranian Society of Regional Anesthesia and Pain Medicine(ISRAPM). This is an open-access article distributed under the terms of the Creative Commons Attribution-NonCommercial 4.0 International License (http://creativecommons.org/licenses/by-nc/4.0/) which permits copy and redistribute the material just in noncommercial usages, provided the original work is properly cited. 
they cannot include or exclude the diagnosis of CRPS. Stellate ganglion blocks are of use in treating the sympathetic part of CRPS upper extremity pain and lumbar sympathetic block is of use in treating the sympathetic part of CRPS lower extremity pain. An indwelling sympathetic-chain catheter, once effective, provides continuous pain relief, while not motor or sensory dysfunction and may be extremely effective in permitting PT to proceed. Single-shot sympathetic blocks ought to be coordinated with PT sessions, so the patient is painless throughout the sessions.

Although lumbar sympathetic blockade has often maintained to treat CPRS, the technique of such blockade is more difficult and not like a peripheral nerve blockade, usually not within the repertoire of active pediatric anesthesiologists. A brachial plexus blockade is of use in treating the somatic part of CRPS upper extremity pain. Different approaches to the current block exist including the supraclavicular block, interscalene block, axillary block and the infraclavicular block with the latter being the foremost common. Continuous popliteal blocks, sciatic peripheral nerve block and saphenous peripheral nerve blockade are of use in treating the somatic part of CRPS lower extremity pain. Continuous epidural analgesia permits a similar level of pain management for PT. Peripheral nerve catheters provide the advantage over continuous epidural infusions and are applicable in upper extremity disease, provide unilateral analgesia, give a restricted and localized sympathetic blockade and do not have an effect on bladder or bowel function. Nevertheless, continuous peripheral nerve block ought to be easier at home than continuous epidural analgesia. Using disposable pumps permissible reduced hospital stay and continuing this treatment at home (e.g. $0.1 \mathrm{~mL} / \mathrm{kg} /$ hour continuous infusion of ropivacaine $0.2 \%$ ). Indwelling epidural catheters, though usually effective in relieving the pain, caused spare motor and/or sensory block that the patient cannot effectively participate in PT. This might be harmful as any immobilization of the limb seems to worsen CRPS. For those who cannot sustain im- provement, despite associate current exercise program, neurosurgical techniques like SCS or in extremely elite cases, sympathectomy is helpful (4-6). Although several modalities are tried for the treatment of CRPS, given the debilitating nature of disorder and variable response to medical aid, new and different therapeutic interventions like continuous peripheral nerve catheters are necessary. For using such techniques, adherence to local anesthetic guidelines is necessary to avoid toxicity. Though continuous peripheral nerve catheters reduce pain and facilitate intensive PT and practical rehabilitation, they resulted in resolution of physical changes associated with CRPS, and a decrease need for pharmacological drugs including opioids. Nonetheless, current care regimens target interruption of disease process instead of symptom management. Regional techniques are useful to improve fast relief; however, do not address the crux of the matter. There is insufficient data to allow any conclusions regarding the effects of continuous peripheral nerve blockade (7). Regional nerve blocks are also of importance in some cases; however, they are not first-line treatment within the care of youngsters with CRPS.

\section{References}

1. Binder A, Schattschneider J, Baron R. Complex Regional Pain Syndrome Type I (Reflex Sympathetic Dystrophy). In: Waldman SD editor. Pain management.. Philadelphia: Saunders; 2007. p. 283.

2. Stanton-Hicks M. Plasticity of complex regional pain syndrome (CRPS) in children. Pain Med.2010;11(8):1216-23.

3. Wilder RT. Management of pediatric patients with complex regional pain syndrome. Clin J Pain. 2006;22(5):443-8.

4. Martin DP, Bhalla T, Rehman S, Tobias JD. Successive multisite peripheral nerve catheters for treatment of complex regional pain syndrome type I. Pediatrics. 2013;131(1):e323-6.

5. Margic K, Pirc J. The treatment of complex regional pain syndrome (CRPS) involving upper extremity with continuous sensory analgesia. Eur J Pain. 2003;7(1):43-7.

6. Dadure C, Motais F, Ricard C, Raux O, Troncin R, Capdevila X. Continuous peripheral nerve blocks at home for treatment of recurrent complex regional pain syndrome I in children. Anesthesiology. 2005;102(2):387-91.

7. Petje G, Radler C, Aigner N, Walik N, Kriegs Au G, Grill F. Treatment of reflex sympathetic dystrophy in children using a prostacyclin analog: preliminary results. Clin Orthop Relat Res. 2005(433):178-82. 